\title{
Long Non-coding RNAs: Regulators of the Activity of Myeloid-Derived Suppressor Cells
}

\author{
Gabriela Leija Montoya ${ }^{1}$, Javier González Ramírez ${ }^{2}$, Jorge Sandoval Basilio ${ }^{3}$, \\ Idanya Serafín Higuera ${ }^{4}$, Mario Isiordia Espinoza ${ }^{5}$, Rogelio González González ${ }^{6}$ and \\ Nicolás Serafín Higuera ${ }^{7 *}$
}

${ }^{1}$ Facultad de Medicina, Universidad Autónoma de Baja California, Mexicali, Mexico, ${ }^{2}$ Facultad de Enfermería, Universidad Autónoma de Baja California, Mexicali, Mexico, ${ }^{3}$ Laboratorio de Biología Molecular, Universidad Hipócrates, Acapulco de Juárez, Mexico, ${ }^{4}$ Facultad de Medicina, Universidad Autónoma de Baja California, Tijuana, Mexico, ${ }^{5}$ División de Ciencias Biomédicas, Departamento de Clínicas, Centro Universitario de los Altos, Universidad de Guadalajara, Tepatitlán de Morelos, Guadalajara, Mexico, ${ }^{6}$ Departamento de Investigación, Universidad Juárez del Estado de Durango, Durango, Mexico,

${ }^{7}$ Unidad de Ciencias de la Salud, Facultad de Odontología, Universidad Autónoma de Baja California, Mexicali, Mexico

\section{OPEN ACCESS}

Edited by: Carlo Pucillo,

University of Udine, Italy

Reviewed by:

Giacomo Desantis,

San Raffaele Hospital (IRCCS), Italy

Shengjun Wang,

Jiangsu University, China

Patrizia Scapini,

University of Verona, Italy

*Correspondence:

Nicolás Serafín Higuera nserafin@uabc.edu.mx

Specialty section

This article was submitted to

Molecular Innate Immunity,

a section of the journal

Frontiers in Immunology

Received: 16 February 2019

Accepted: 09 July 2019

Published: 25 July 2019

Citation:

Leija Montoya G, González Ramírez J,

Sandoval Basilio J, Serafín Higuera I,

Isiordia Espinoza M, González González $R$ and Serafín Hiquera N

(2019) Long Non-coding RNAs:

Regulators of the Activity of

Myeloid-Derived Suppressor Cells.

Front. Immunol. 10:1734.

doi: 10.3389/fimmu.2019.01734
Myeloid-derived suppressor cells (MDSCs) are a heterogeneous cell population with potent immunosuppressive functions. They play major roles in cancer and many of the pathologic conditions associated with inflammation. Long non-coding RNAs (IncRNAs) are untranslated functional RNA molecules. The IncRNAs are involved in the control of a wide variety of cellular processes and are dysregulated in different diseases. They can participate in the modulation of immune function and activity of inflammatory cells, including MDSCs. This mini review focuses on the emerging role of IncRNAs in MDSC activity. We summarize how IncRNAs modulate the generation, recruitment, and immunosuppressive functions of MDSCs and the underlying mechanisms.

Keywords: myeloid-derived suppressor cell, long non-coding RNA, inflammation, cancer, immunosuppressive function, accumulation

\section{INTRODUCTION}

The chronic inflammatory conditions typically observed in many diseases can promote the accumulation of myeloid-derived suppressor cells (MDSCs) (1). This heterogeneous cell population with a strong immunosuppressive function has been principally studied in cancer. However, in recent years, the role of MDSCs has been assessed in other conditions, such as diabetes mellitus, obesity, autoimmune diseases, and infectious diseases (2-4).

Different subsets of MDSCs have been reported; in mice, monocyte MDSCs (M-MDSCs) are described as cluster of differentiation (CD) $11 \mathrm{~b}^{+} \mathrm{Ly}_{6 \mathrm{G}}{ }^{-} \mathrm{Ly} 6 \mathrm{C}^{+}$cells, and

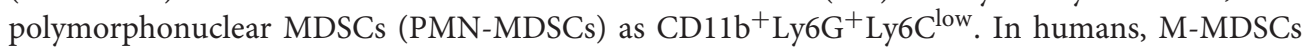
are characterized as $\mathrm{CD} 11 \mathrm{~b}^{+} \mathrm{CD} 33^{+} \mathrm{HLADR}^{-} \mathrm{CD} 14^{+}$, whereas PMN-MDSCs are defined as $\mathrm{CD}_{11 b^{+}} \mathrm{CD}_{3} 3^{+} \mathrm{HLADR}^{-} \mathrm{CD} 15^{+}(5)$. However, other phenotypes have been described in different tumors and infectious diseases $(2,4,6)$.

In addition to their high heterogeneity, MDSCs present functional heterogeneity (2). The immunoregulatory functions of MDSCs include the generation of immunosuppressive cells (e.g., regulatory $\mathrm{T}$ cells and $\mathrm{M} 2$ macrophages) by the production of interleukin (IL)-10; the production of reactive oxygen species (ROS) using the isoforms of nicotinamide adenine dinucleotide phosphate (NADPH) oxidase (NOX1, NOX2, NOX3, and NOX4); 
and production of reactive nitrogen species, predominantly nitric oxide (NO), by the activation of inducible nitric oxide synthase (iNOS). These reactive species can inhibit the proliferation of $\mathrm{T}$ cells, induce the apoptosis of $\mathrm{T}$ cells, and reduce both the expression of the $\zeta$ chain of T-cell receptors (TCRs), as well as TCR nitration. Moreover, NO can induce the expression of cyclooxygenase 2 (COX2), which regulates the production of prostaglandin-E2, an important molecule that promotes the upregulation of IL-10 and arginase-1 (Arg-1) expression (1). In addition, MDSCs impair the metabolic functions and proliferation of $\mathrm{T}$ cells by producing Arg-1, NOS, arginineglycine amidinotransferase, and L-arginine decarboxylase. The MDSCs can express regulatory molecules, such as programmed death-ligand-1 and Fas ligand to induce the anergy and apoptosis of $\mathrm{T}$ cells (1).

The MDSCs originate from common myeloid progenitors in the bone marrow. In addition, extramedullary myelopoiesis in pathologic conditions can generate MDSCs $(7,8)$. The chronic inflammatory stimuli generated in cancer or infections can induce "emergency myelopoiesis," which is characterized by the expansion of immature myeloid cells to counterbalance the loss of cells $(9,10)$. Previously, a "two-signal" model of MDSC accumulation was suggested (11), in which the expansion of immature myeloid cells would be supported by growth factors, such as granulocyte-macrophage colonystimulating factor, granulocyte colony-stimulating factor, and macrophage colony-stimulating factor. However, a "first signal" would also be required to maintain these cells in an undifferentiated state. A "second signal" would promote the activation of immunosuppressive functions, and thereby generate MDSCs (11).

Transcription factors, such as signal transducer and activator of transcription (STAT)3, interferon regulatory factor-8, retinoblastoma protein $(\mathrm{RB}) 1$, and CCAAT/enhancer binding protein $(\mathrm{C} / \mathrm{EBP}) \beta$ have been linked to the first signal. Stimulation of the immunosuppressive program has been related, for example, to the activation of the transcription factor, nuclear factor-kappa B (NF-kB) through myeloid differentiation factor (MyD)88, the activation of the STAT1 and STAT6 pathways, and endoplasmic reticulum stress-related pathways associated with the transcription factor, C/EBP homologous protein (CHOP) $(11,12)$. In addition, the factors associated with the two signals could overlap $(11,12)$. For example, C/EBP $\beta$, a member of the C/EBP leucine zipper domain-containing family, has three isoforms because of different initiation codons: the liver-inhibitory protein (LIP), liver-activating protein (LAP), and full-length liver-activating protein $\left(\mathrm{LAP}^{*}\right)$. The LAP and LAP* have been considered transcription activators, whereas LIP is considered a repressor or a dominant negative inhibitor of other C/EBP family members $(13,14)$. Furthermore, C/EBP $\beta$ is involved in the regulation of "emergency granulopoiesis" generated by infections or cytokines (15). McPeak et al. showed that reduced expression of $\mathrm{C} / \mathrm{EBP} \beta$ in the myeloid cells of conditional knockout mice was associated with reduced accumulation of MDSCs in a polymicrobial sepsis model (16). In addition, when all CEBP $\beta$ isoforms were deleted in hematopoietic lineage cells using tumor mouse models, MDSC accumulation was diminished. Moreover, the MDSCs of these mice showed reduced activity and production of Arg-1 and iNOS proteins (14). In vitro studies have suggested that the LAP isoform can bind specific sequences in the regulatory regions of Arg-1, COX2, NOX2, and $i N O S$, and promote their expression in MDSCs. In addition, LIP can supposedly interact with LAP to inhibit its transcription function $(17,18)$. Thus, C/EBP $\beta$ could regulate the expansion and suppression of MDSCs.

The MDSCs have been considered to be central regulators in tumor microenvironments. Elimination of MDSCs by targeting the pathways or molecules involved in their generation, expansion, activation, or recruitment at distant sites, and immunosuppressive function in the local microenvironment could improve the response to cancer treatment (19). Thus, a deeper understanding of the mechanisms involved in the control of these processes is important.

In this context, the epigenetic regulation of the biologic behavior of MDSCs has emerged as a novel field and promising tool in therapy (20). "Epigenetics" refers to heritable changes without variations in DNA sequences, and the study of chromatin (21). Thus, epigenetic mechanisms analyzed in MDSCs involve DNA methylation, histone modifications, and regulation by non-coding RNAs $(2,20,22)$. In this review, we summarize current knowledge about the central role of long non-coding RNAs (lncRNAs), a type of non-coding RNA that modulates the generation, recruitment, and immunosuppressive function of MDSCs (Table 1). In addition, the underlying molecular mechanisms will be described in some cases.

\section{IncRNAs}

The lncRNAs are transcripts larger than 200 nucleotides without coding capacity (31). It has been predicted that the human genome encodes $>28,000$ lncRNAs, most of which are uncharacterized (32). The biogenesis of IncRNAs has several similarities with the biogenesis of messenger RNA (mRNA) (33). In most cases, lncRNA production is carried out by RNA polymerase II. Modifications include the elimination of introns and the addition of a poly- $\mathrm{A}$ tail at the $3^{\prime}$ end, and many (but not all) IncRNAs have a cap in their structure at the $5^{\prime}$ end (33-35).

Based on their genomic localization, IncRNAs can be classified as "intronic" (encoded in the introns of genes), "intergenic" (encoded in the regions between two genes), "enhancer" (encoded in the regions of enhancer promoters), "bidirectional" (encoded in the vicinity of a gene of the opposite strand), "senseoverlapping" (encoded in the introns and exons of different genes in the sense strand of DNA), and "antisense" (encoded in the antisense strands of DNA) (32).

The lncRNAs are highly heterogeneous and have substantial functional versatility based on their capacity to be adapted to different structures and molecular interactions (36). In the nucleus, lncRNAs can act as regulators of transcription (regulating DNA methylation, joining transcription factors, and modifying chromatin); be involved in RNA processing (by means of splicing and antisense alignment); act as "molecular decoys" for proteins, such as p53; and be precursors of microRNAs 
TABLE 1 | LncRNAs and their mechanisms implicated in the accumulation and function of myeloid-derived suppressor cells (MDSCs).

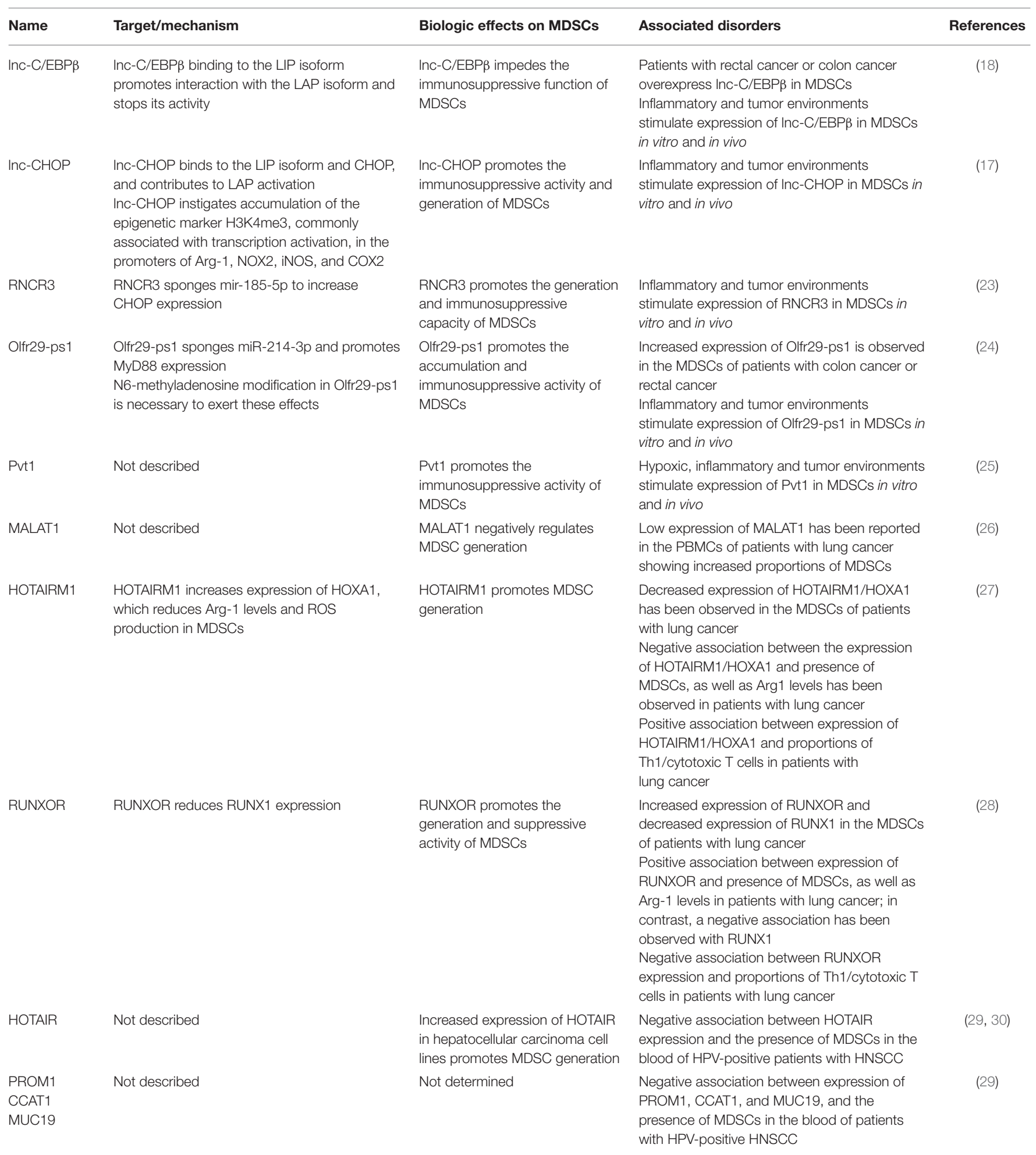

(miRNAs). In the cytoplasm, they can act as molecular decoys for miRNAs, and "scaffolds" for cytoplasmic proteins (37).

Although only a small proportion of all identified lncRNAs have been studied in depth, they are fundamental in many cellular contexts and diverse biological processes (38). In this context, these functional transcripts regulate the differentiation of megakaryocytes, granulocytes, monocytes, and macrophages, and modulate the inflammatory response $(39,40)$. 


\section{EXPRESSION AND FUNCTION OF LNCRNAS IN MDSCS \\ Inc-C/EBP $\beta$}

The lnc-C/EBP $\beta$ (also named E130102H24Rik) is an intergenic lncRNA conserved in mice and humans that is encoded in chromosome 1 and chromosome 4, respectively. In addition, it has been found predominantly in cellular nuclei. High expression of lnc- $\mathrm{C} / \mathrm{EBP} \beta$ in the myeloid cells (e.g., macrophages and dendritic cells) of mice has been reported. Importantly, differential expression of $\operatorname{lnc}-\mathrm{C} / \mathrm{EBP} \beta$ has been observed in mouse MDSCs if variations in the inflammatory environment occur. Moreover, IL-6 can promote lnc- $\mathrm{C} / \mathrm{EBP} \beta$ expression in MDSCs. Expression of lnc-C/EBP $\beta$ has also been reported in M-MDSCs, as well as the PMN-MDSCs of patients with colon cancer or rectal cancer (18). The lnc-C/EBP $\beta$ can inhibit expression of enzymes, such as Arg-1, iNOS, NOX2, and COX2, in mouse MDSCs and human MDSC-like cells, resulting in lower concentrations of their metabolic products.

Overexpression of $\operatorname{lnc} \mathrm{C} / \mathrm{EBP} \beta$ in MDSCs promotes the expression of interferon (IFN) $-\gamma$ in T cells. Tumors in murine models show slower growth if mice are treated with MDSCs overexpressing lnc-C/EBP $\beta$, and tumor-infiltrating $\mathrm{T}$ cells demonstrate increased expression of IFN- $\gamma$, as compared with controls (18). Thus, lnc-C/EBP $\beta$ can attenuate the immunosuppressive function of MDSCs. The suggested mechanism by which this is achieved is the binding of lnc$\mathrm{C} / \mathrm{EBP} \beta$ to $\mathrm{C} / \mathrm{EBP} \beta$ (specifically to the LIP isoform), which promotes the interaction of LIP with the transcription activator LAP. These interactions prevent the accumulation of LAP in the promoters of Arg-1, iNOS, NOX2, and COX2, resulting in decreased expression of these enzymes (18).

Expression of lnc-C/EBP $\beta$ can block the generation of murine M-MDSCs (18). Because lnc-C/EBP $\beta$ has conserved expression and negatively regulates the differentiation and immunosuppressive activity of MDSCs, it could be a potential target for future studies in immunotherapy. To our knowledge, only one report has analyzed this lncRNA. Hence, further studies on the additional functions of lnc-C/EBP $\beta$ in various physiological processes and cancer, as well as the molecular mechanisms involved in its regulation of the immune response are necessary.

\section{Inc-CHOP}

The lnc-CHOP (also named GM16727) is an intronic lncRNA that has not been widely characterized. It is encoded in chromosome 11 and localized in cellular nuclei. Interleukins, such as IL-6 and tumor necrosis factor (TNF)- $\alpha$, and tumorassociated factors can induce the increased expression of lnc-CHOP in mouse MDSCs. Overexpression of lnc-CHOP promotes the expression of Arg-1, NOX2, iNOS, and COX2 and their metabolites in MDSCs, and contributes to the reduction of IFN- $\gamma$ produced by T cells (17). Thus, lnc-CHOP fosters the immunosuppressive activity of mouse MDSCs.

Furthermore, lnc-CHOP positively regulates MDSC generation and promotes tumor growth in murine models. It has been suggested that lnc-CHOP binds to the transcription factor CHOP and the C/EBP $\beta$ isoform, LIP. This allows the activation of LAP and its accumulation in the promoters of target genes, thereby promoting the expression of Arg-1, NOX2, NOS2, and COX2. In addition, increased expression of these enzymes could be the result of enrichment of trimethylation of the amino acid, lysine, at position 4 in the histone $\mathrm{H} 3$ (H3K4me3) marker of their promoter regions. The $\mathrm{H} 3 \mathrm{~K} 4 \mathrm{me} 3$ marker is usually enriched at active chromatin regions and its accumulation is promoted by overexpression of lnc-CHOP in MDSCs (17). The lnc-CHOP could use different mechanisms to promote the accumulation and activation of MDSCs. The conservation and role of lnc-CHOP in human MDSCs, as well as its contribution in tumor biology, has yet to be determined. Future studies could ascertain the additional functions of lnc-CHOP and its potential applications.

\section{Retinal Non-coding RNA (RNCR)3}

The RNCR3 is an intergenic lncRNA that is highly conserved in mammals (in which it is also known as LINC00599) (41). The RNCR3 expression is reportedly related to glioblastoma, prostate cancer, atherosclerosis, and retinal microvascular abnormalities (41-44). Mouse MDSCs express nuclear and cytoplasmic RNCR3, the expression of which is increased in mice with tumors. In addition, IL- 6 induces the increased expression of this lncRNA in the MDSCs of mice (23). Downregulation of RNCR3 expression prevents MDSC differentiation in vitro and in vivo, whereas RNCR3 expression promotes the preferential differentiation of PMN-MDSCs. Importantly, RNCR3 contributes to the immunosuppressive function of MDSCs to induce the expression of Arg-1 and iNOS in vitro.

Furthermore, IFN- $\gamma$ production by $\mathrm{T}$ cells is increased in the presence of MDSCs with reduced expression of RNCR3. A tumor model in mice treated with MDSCs down-regulating the expression of RNCR3 showed increased tumoral growth (23). One possible mechanism for this is the use of RNCR3 to "sponge" mir-185-5p. The latter impedes MDSC generation and the production of INOS and Arg-1 by targeting CHOP. In the presence of RNCR3, mir-185-5p binds to it preferentially, resulting in the upregulation of CHOP expression (23). The immunosuppressive function of MDSCs is promoted by $\mathrm{CHOP}$, as CHOP-deficient MDSCs show increased expression of the LIP isoform and reduced binding of $\mathrm{C} / \mathrm{EBP} \beta$ to promoters of Arg1 and IL-6. This results in the reduced expression of IL-6 and activation of STAT3, as well as impaired immunosuppressive function (45). Thus, RNCR3 supports the accumulation and immunosuppressive program of MDSCs. Additional studies could determine whether RNCR3 exerts biological effects on human MDSCs for potential applications against chronic inflammatory diseases in humans.

\section{Olfactory Receptor 29, Pseudogene 1 (Olfr29-ps1)}

The Olfr29-ps1 is a lncRNA in mice (OR1F2P in humans) that has not been characterized previously. It is conserved and expressed in the nuclei and cytoplasm of murine and human MDSCs and macrophages. Tumor-associated factors and IL-6 can increase the expression of Olfr29-ps1 in the MDSCs of mice. 
Mononuclear cells with a MDSC phenotype in patients with colon cancer or rectal cancer have shown increased expression of Olfr29-ps1 (24). The overexpression of Olfr29-ps1 in bone marrow cells (BMCs) has been shown to promote the generation of mouse M-MDSCs in a differentiation model using cellular cultures, and an in vivo BMC chimera model. In addition, augmented accumulation of M-MDSCs has been observed in the tumors and spleen of a tumor model in mice with Olfr29ps1-overexpressing MDSCs. The immunosuppressive activity of human and murine MDSCs is increased by Olfr29-ps1 overexpression (24).

In vitro analysis showed that IFN- $\gamma$ production by $\mathrm{T}$ cells is reduced in the presence of Olfr29-ps1-overexpressing MDSCs, and that these MDSCs show increased protein expression of Arg1, COX2, NOX2, and iNOS, as well as increased production of their metabolites. Tumors in a mouse model with Olfr29ps1-overexpressing MDSCs show greater growth and fewer infiltrating $\mathrm{T}$ (especially $\mathrm{CD}^{8+}$ ) cells. In vitro analyses suggest that the effects generated by Olfr29-ps1 could be explained (at least in part) by its capacity to sponge miR-214-3p (24). The latter inhibits the expression of the mRNA and protein expression of MyD88, so interactions between Olfr29-ps1 and miR-214-3p result in the augmented expression of MyD88 (24). Thus, the immunosuppressive activity of MDSCs is promoted by MyD88.

Interestingly, modification of N6-methyladenosine in regions of the Olfr29-ps1 sequence is essential for the stability and function of Olfr29-ps1 in MDSCs. This modification is common in mRNAs and generated by methyltransferases, such as methyltransferase-like (METTL)3. Notably, the downregulation of METTL3 expression reduces Olfr29-ps1 production, as well as the immunosuppressive activity and generation of MDSCs (24). Mechanistically, these observations are important because modification of N6-methyladenosine is reversible and could have potential therapeutic benefits. However, only additional research will show whether this is possible.

\section{Plasmacytoma Variant Translocation (Pvt)1}

The Pvt1 is an intergenic lncRNA. It is conserved in humans and mice. Notably, it is over-expressed in several human cancers, including melanoma, cervical cancer, gastric cancer, prostate cancer, hepatocellular cancer, esophageal cancer, and acute myeloid leukemia $(25,46,47)$. Tumor-infiltrating PMN-MDSCs and M-MDSCs show increased expression of Pvt1 in tumor mouse models, and overexpression in the splenic MDSCs of those mice. In addition, the presence of IL-6 increases the expression of Pvt1 in PMN-MDSCs generated in cultures. Interestingly, hypoxic conditions and expression of hypoxia-inducible factor (HIF)- $1 \alpha$ increases Pvt1 production in PMN-MDSCs in vitro (25). Thus, inflammatory and tumor microenvironments could promote the increased expression of this lncRNA in MDSCs.

Downregulation of Pvt1 expression in PMN-MDSCs can induce reduced production of ROS and Arg-1 activity, as well as a slight increase in T-cell proliferation in co-cultures. A tumor mouse model treated with Pvt1-down-regulating PMNMDSCs showed reduced growth in the generated tumors and a modest increase in the number of CD8 $+\mathrm{T}$ cells producing IFN- $\gamma$ in lymphatic nodules (25). These results suggest that
Pvt1 promotes immunosuppressive activity in PMN-MDSCs. Whether Pvt1 modulates the immunosuppressive functions of human MDSCs warrants future exploration. In addition, the molecular mechanisms involved in MDSC regulation by this lncRNA should be investigated.

Interestingly, similar microenvironmental factors (e.g., IL-6 or tumor-associated factors) can induce the overexpression of lncC/EBP $\beta$, lnc-CHOP, Olfr29-ps1, Pvt1, and RNCR3 in MDSCs. These microenvironmental factors produce contrasting effects because lnc-CHOP, Olfr29-ps1, Pvt1, and RNCR3 promote, whereas lnc- $\mathrm{C} / \mathrm{EBP} \beta$ prevents, the immunosuppressive functions and differentiation of MDSCs (17, 18, 23-25). These actions could indicate "fine tuning" of gene regulation and the importance of lncRNAs in the control of the biological behavior of MDSCs. In addition, the final biological effect could result in crosstalk among the diverse pathways regulated by lncRNAs.

\section{Metastasis-Associated Lung Adenocarcinoma Transcript (MALAT)1}

The MALAT1 (also named nuclear-enriched abundant transcript-2) is a nuclear intergenic lncRNA. It is highly conserved among species and involved in various diseases. The MALAT1 is considered an oncogene because it can promote the proliferation, invasion, and metastasis of many types of human cancer cells (48). Thus, this lncRNA has been studied to develop new strategies in the diagnosis and treatment of cancer (48). Using an in vitro differentiation model, Zhou and colleagues recently reported that the reduced expression of MALAT1 in peripheral blood mononuclear cells (PBMCs) promotes their differentiation to MDSC-like cells. Interestingly, the reduced expression of MALAT1 has been reported in the PBMCs of patients with lung cancer, as well as an increased proportion of MDSCs (26). Thus, MALAT1 could negatively regulate the differentiation of MDSCs in patients with lung cancer. More studies evaluating the role and mechanisms through which MALAT1 regulates MDSC differentiation in different diseases could lead to new directions in potential therapeutics.

\section{HOXA Transcript Antisense RNA Myeloid-Specific (HOTAIRM)1}

The HOTAIRM1 is an intergenic lncRNA localized between homeobox $(H O X) A 1$ and HOXA2 genes, and is expressed preferentially in the myeloid lineage. It has been associated with glioblastoma and myeloid leukemia, as well as colorectal, pancreatic, lung, and breast cancer (49, 50). Importantly, HOTAIRM1 regulates the differentiation of myeloid cells (27, 50 ). Reduced expression of HOTAIRM1 has been reported in the MDSCs of tumors of patients with lung cancer. In addition, the overexpression of HOTAIRM1 has been shown to reduce the differentiation of MDSCs and production of Arg-1 in cellular cultures using human PBMCs (27). Consistent with those data, diminished expression of HOTAIRM1 was found in the PBMCs of patients with lung cancer, as well as increased proportions of MDSCs. Moreover, a negative association was observed between HOTAIRM1 expression and the presence of MDSCs, as well as Arg-1 production; whereas a positive association was observed 
with respect to the percentage of T-helper (Th)1 cells and cytotoxic T cells in the same patients (27).

The mechanism by which HOTAIRM1 regulates MDSCs could be associated with HOXA1 expression. The HOTAIRM1 can induce HOXA1 expression in MDSCs, which reduces Arg- 1 expression and ROS production. In addition, increased expression of HOXA1 has been shown to reduce tumor growth, decrease the percentage of MDSCs, and enhance the immune response in a tumor mouse model. Moreover, a positive association has been observed between the expression of HOTAIRM1 and HOXA1 in patients with lung cancer (27). These observations suggest that HOTAIRM1 inhibits the differentiation and suppressive activity of human and mouse MDSCs. Further studies analyzing the effects of HOTAIRM1 on MDSCs in other tumor types should be conducted. In addition, the mechanisms involved in the reduced expression of HOTAIRM1 in lung cancer have yet to be determined.

\section{Runt-Related Transcription Factor-1 Overlapping RNA (RUNXOR)}

The RUNXOR is an intragenic lncRNA that has been very rarely studied. It is localized in the locus of the runt-related transcription factor $(R U N X) 1$ gene, and its expression is increased in the bone marrow of patients with acute myeloid leukemia (51). The MDSCs generated in cell cultures using the PBMCs and MDSCs of tissue from patients with lung cancer express high levels of RUNXOR. Furthermore, the downregulated expression of this IncRNA in PBMCs disturbs their differentiation to MDSCs in a cell-culture model. Moreover, Arg1 expression is reduced if RUNXOR expression is decreased in MDSCs. Thus, RUNXOR is involved in promoting the generation and immunosuppressive function of MDSCs (28).

Interestingly, the increased expression of RUNXOR has been reported in the PBMCs of patients with lung cancer, and a positive correlation has been described between RUNXOR expression and the presence of MDSCs, as well as Arg-1 production in such patients. In contrast, a negative correlation has been observed between RUNXOR expression and the percentage of both Th1 cells and cytotoxic $\mathrm{T}$ cells (28). It has been suggested that RUNXOR exerts its biologic effects on MDSCs through its target RUNX1. Wang and co-workers suggested that RUNXOR binds the enhancer of zeste homolog 2 (histone H3K27 methyltransferase component of polycomb repressive complex 2) and RUNX1 protein to the RUNX1 promoter; and the RUNXOR promoter could compete with the RUNX1 promoter for the transcription machinery (51). Thus, RUNXOR could reduce RUNX1 expression in MDSCs in vitro and in patients with lung cancer. Reduced expression of RUNX1 in mouse MDSCs promotes the production of Arg1 , iNOS, and ROS in vitro. Moreover, RUNX1 expression induces the differentiation of MDSCs into myeloid cells with a mature phenotype (52). Therefore, RUNXOR could promote the expansion and immunosuppressive activity of MDSCs in lung cancer.

Evidently, MALAT1, HOTAIRM1, and RUNXOR regulate important biological activities (e.g., expansion, differentiation, and immunosuppressive functions) of MDSCs in lung cancer. Hence, these lncRNAs could offer opportunities for potential therapeutic applications against lung cancer; nevertheless, a considerable amount of research would be necessary.

\section{Hox Antisense Intergenic RNA (HOTAIR)}

The HOTAIR is an oncogenic lncRNA positively associated with initiation, growth, angiogenesis, progression, drug resistance, and poor prognosis in cancer (53). The expression of HOTAIR has been indirectly related to MDSC recruitment. A negative association has been reported between HOTAIR expression and the proportion of MDSCs in the blood samples of patients with human papillomavirus-positive head and neck squamous cell carcinoma (HPV-positive HNSCC) (29), but a causal relationship has not been established. Furthermore, HOTAIR overexpression in cell lines of hepatocellular carcinoma can induce increased production of the $\mathrm{C}-\mathrm{C}$ motif chemokine ligand (CCL)2 (30). The CCL2 is not a specific chemokine for MDSCs and is a chemoattractant for several tumor-related myeloid cells (including monocytes and tumor-associated macrophages). Hence, HOTAIR expression could exert a more generalized function by promoting inflammation and immunosuppression within the tumor microenvironment. In addition, using a differentiation model of PBMCs from human donors, cell lines of hepatocellular carcinoma overexpressing HOTAIR promoted MDSC differentiation in co-cultures (30). Thus, HOTAIR expressed by tumor cells could positively regulate MDSC generation in vitro; nevertheless, the associated molecular mechanisms have not been determined. Studies are needed to ascertain the functional role of HOTAIR in the recruitment or differentiation of MDSCs.

\section{Other IncRNAs}

In addition to HOTAIR, three other lncRNAs have been negatively associated with MDSCs in the blood samples of patients with HPV-positive HNSCC: prominin (PROM)1, colon cancer associated transcript (CCAT), and mucin (MUC) 19 (29). However, the molecular mechanisms implicated in the recruitment or expansion of MDSCs have not been determined, because a direct molecular role of these IncRNAs in MDSC biology has not been reported. In future studies, these lncRNAs could be evaluated as potential biomarkers in patients with HPVpositive HNSCC, because the proportion of MDSCs is increased in these patients as compared with precancerous lesions and normal oral mucous tissues (29).

To our knowledge, only one study has focused on the relationship between lncRNAs and MDSCs in non-cancerrelated diseases. The IncRNA expression was analyzed in the MDSCs generated in mice infected with Echinococcus granulosus (54) (the causal agent of cystic echinococcosis in humans). This zoonotic disease principally affects the liver and lungs. In mice infected with E. granulosus, the expansion of MDSCs that down-regulate the activity of $\mathrm{T}$ cells has been reported (55). These MDSCs, in the presence of this infectious agent, showed 649 differentially expressed lncRNAs. Bioinformatics analyses based on mRNA expression revealed alterations in biologic processes (e.g., signaling by mechanistic target 
of rapamycin) and the involvement of 288 lncRNAs in the cis-regulation of their sense-overlapping genes. Interestingly, $R b 1$ regulation by the IncRNA NONMMUT021591s was predicted; 60 transcription factors regulating expression of $372 \operatorname{lncRNAs}$ predicted the regulation of the lncRNA, FR015378, by C/EBP $\beta$ (54). Additional studies could determine the biological contribution of lncRNAs in modulating the function and differentiation of MDSCs in the context of infections.

\section{CONCLUSIONS AND FUTURE PERSPECTIVES}

Studies have suggested that specific IncRNAs control the differentiation of MDSC subsets and immunosuppressive function. Furthermore, lncRNAs show specific patterns of expression depending on the cell and tissue types (39). Thus, IncRNAs could be potential specific markers of MDSC subsets and several MDSCs with diverse phenotypes that have been observed in various diseases, but these have yet to be determined. In addition, controversy has been generated because the close relationship among MDSCs, neutrophils, and monocytes. Analyzing the expression profile of lncRNAs in MDSCs compared with that of lncRNAs in myeloid cells could provide new insights into the differences described among these cell types. Studies analyzing lncRNAs exclusively in mouse MDSCs should be viewed with caution, considering that IncRNAs seem to be poorly conserved among various species (56). Therefore, investigations into the modulation of MDSC activity by lncRNAs should consider the evolutionary conservation of lncRNA in human MDSCs for potential applications against human diseases. The lncRNA expression profile in human MDSCs has yet to be reported.

Understanding the regulation of immunosuppressive function and the accumulation of MDSCs to find therapeutic targets that modulate immunosuppression is more important than the classification of MDSCs among myeloid cells. The lncRNAs regulate the activity of different transcription factors (e.g., $\mathrm{C} / \mathrm{EBP} \beta, \mathrm{CHOP}$, and RUNX1) involved in the differentiation and suppression of MDSCs. Thus, these non-coding RNAs might play significant roles in the two-signal model (11), in which lncRNAs (such as Olfr29-ps1, lnc-CHOP, RNCR3, and RUNXOR) could participate in the first phase during expansion of the MDSCs, and then in the second phase to promote MDSC activation. The Pvtl could participate only in the second phase. The versatility of lncRNAs in the recognition of different targets could facilitate their participation in both phases. In addition, the factors produced by tumors, hypoxia, or an inflammatory microenvironment could support the expression of some non-coding RNAs. Moreover, the chronic and low-dose stimuli generated by inflammatory and tumoral factors could promote the downregulation of lncRNAs that inhibit the accumulation or immunosuppressive function of MDSCs. Future studies will determine whether this perception is correct.
In addition to intracellular regulation, $\operatorname{lncRNAs}$ can exert intercellular effects via exosomes $(57,58)$. These extracellular nanovesicles are derived from endosomes, have a diameter of $30-100 \mathrm{~nm}$, and are secreted by different cell types, including cells with a myeloid lineage, such as MDSCs $(59,60)$. Limited information on MDSC exosomes indicates that they can exert effects associated with immunosuppression and the promotion of tumorigenesis (61). The MDSC exosomes carry proteins, RNAs, and miRNAs (62), but the presence of IncRNAs in MDSC exosomes has not been investigated. Future studies addressing this issue would be important, because lncRNAs are supposedly selectively packaged in exosomes and secreted by cancer cells and stroma cells to modulate the growth, metastasis, angiogenesis, and chemoresistance of cancer cells $(57,58)$. Moreover, the characterization of lncRNAs in exosomes secreted by myeloid lineage cells is not widely understood.

The lncRNAs involved in the biological behavior of MDSCs could facilitate the development of novel therapeutic approaches. However, if these lncRNAs are involved in multiple physiological functions or have contrasting effects in different cell types, then alteration/manipulation of lncRNAs could also generate undesirable side effects. Thus, one cannot suggest that targeting $\operatorname{lncRNAs}$ is feasible or practical. A more comprehensive understanding of lncRNA functions and the molecular mechanisms implicated in the modulation of MDSCs is necessary.

The central role of MDSCs in generating immunosuppressive tumor microenvironments supports the growth and progression of tumor cells. In addition, a general understanding of the modulation of the inflammatory response by MDSCs in other diseases has been improved in recent years. Thus, molecules that regulate the biological behavior of MDSCs could be the targets of therapies against these diseases.

The lncRNAs are involved in the control of MDSC differentiation and immunosuppressive programs in cancer via various molecular mechanisms (Table 1). Nevertheless, the functional link between some lncRNAs and MDSCs does not seem to be sufficiently strong. Thus, the study of the mechanisms by which lncRNAs modulate MDSCs is in its infancy. The lncRNAs control gene expression and diverse biological functions in health and disease in both cell- and tissue-specific manners. Hence, future studies should aim to identify the novel lncRNAs that regulate MDSC activity, so they can be applied in immunomodulatory therapy or as biomarkers.

\section{AUTHOR CONTRIBUTIONS}

All authors listed have made a substantial, direct and intellectual contribution to the work, and approved it for publication.

\section{FUNDING}

This work was supported by CONACYT grant APN-2015-011650 to GL and Nuevos PTC SEP-PRODEP grant number UDGPTC-1438; 511-6/18/9169 to MI. 


\section{REFERENCES}

1. Groth $\mathrm{C}, \mathrm{Hu} \mathrm{X}$, Weber $\mathrm{R}$, Fleming V, Altevogt $\mathrm{P}$, Utikal J, et al. Immunosuppression mediated by myeloid-derived suppressor cells (MDSCs) during tumour progression. Br J Cancer. (2019) 120:16-25. doi: 10.1038/s41416-018-0333-1

2. Budhwar S, Verma P, Verma R, Rai S, Singh K. The Yin and Yang of myeloid derived suppressor cells. Front Immunol. (2018) 9:2776. doi: 10.3389/fimmu.2018.02776

3. Ma H, Xia CQ. Phenotypic and functional diversities of myeloid-derived suppressor cells in autoimmune diseases. Mediators Inflamm. (2018) 2018:4316584. doi: 10.1155/2018/4316584

4. Penaloza HF, Alvarez D, Munoz-Durango N, Schultz BM, Gonzalez PA, Kalergis AM, et al. The role of myeloid-derived suppressor cells in chronic infectious diseases and the current methodology available for their study. $J$ Leukoc Biol. (2019) 105:857-72. doi: 10.1002/JLB.MR0618-233R

5. Safari E, Ghorghanlu S, Ahmadi-Khiavi H, Mehranfar S, Rezaei R, Motallebnezhad M. Myeloid-derived suppressor cells and tumor: current knowledge and future perspectives. J Cell Physiol. (2019) 234:9966-81. doi: $10.1002 /$ jcp. 27923

6. Solito S, Marigo I, Pinton L, Damuzzo V, Mandruzzato S, Bronte V. Myeloidderived suppressor cell heterogeneity in human cancers. Ann N Y Acad Sci. (2014) 1319:47-65. doi: 10.1111/nyas.12469

7. Millrud CR, Bergenfelz C, Leandersson K. On the origin of myeloid-derived suppressor cells. Oncotarget. (2017) 8:3649-65. doi: 10.18632/oncotarget.12278

8. Salminen A, Kaarniranta K, Kauppinen A. Immunosenescence: the potential role of myeloid-derived suppressor cells (MDSC) in agerelated immune deficiency. Cell Mol Life Sci. (2019) 76:1901-18. doi: 10.1007/s00018-019-03048-x

9. Chiba Y, Mizoguchi I, Hasegawa H, Ohashi M, Orii N, Nagai T, et al. Regulation of myelopoiesis by proinflammatory cytokines in infectious diseases. Cell Mol Life Sci. (2018) 75:1363-76. doi: 10.1007/s00018-017-2724-5

10. Consonni FM, Porta C, Marino A, Pandolfo C, Mola S, Bleve A, et al. Myeloidderived suppressor cells: ductile targets in disease. Front Immunol. (2019) 10:949. doi: 10.3389/fimmu.2019.00949

11. Condamine T, Mastio J, Gabrilovich DI. Transcriptional regulation of myeloid-derived suppressor cells. J Leukoc Biol. (2015) 98:913-22. doi: 10.1189/jlb.4RI0515-204R

12. Gabrilovich DI. Myeloid-derived suppressor cells. Cancer Immunol Res. (2017) 5:3-8. doi: 10.1158/2326-6066.CIR-16-0297

13. Hirai H, Yokota A, Tamura A, Sato A, Maekawa T. Non-steady-state hematopoiesis regulated by the C/EBPbeta transcription factor. Cancer Sci. (2015) 106:797-802. doi: 10.1111/cas.12690

14. Marigo I, Bosio E, Solito S, Mesa C, Fernandez A, Dolcetti L, et al. Tumor-induced tolerance and immune suppression depend on the C/EBPbeta transcription factor. Immunity. (2010) 32:790-802. doi: $10.1016 /$ j.immuni.2010.05.010

15. Hirai H, Zhang P, Dayaram T, Hetherington CJ, Mizuno S, Imanishi J, et al. $\mathrm{C} /$ EBPbeta is required for 'emergency' granulopoiesis. Nat Immunol. (2006) 7:732-9. doi: 10.1038/nil354

16. McPeak MB, Youssef D, Williams DA, Pritchett CL, Yao ZQ, McCall CE, et al. Frontline science: myeloid cell-specific deletion of Cebpb decreases sepsisinduced immunosuppression in mice. J Leukoc Biol. (2017) 102:191-200. doi: 10.1189/jlb.4HI1216-537R

17. Gao Y, Wang T, Li Y, Zhang Y, Yang R. Lnc-chop promotes immunosuppressive function of myeloid-derived suppressor cells in tumor and inflammatory environments. J Immunol. (2018) 200:2603-14. doi: 10.4049/jimmunol.1701721

18. Gao Y, Sun W, Shang W, Li Y, Zhang D, Wang T, et al. LncC/EBPbeta negatively regulates the suppressive function of myeloidderived suppressor cells. Cancer Immunol Res. (2018) 6:1352-63. doi: 10.1158/2326-6066.CIR-18-0108

19. Tesi RJ. MDSC; the most important cell you have never heard of. Trends Pharmacol Sci. (2019) 40:4-7. doi: 10.1016/j.tips.2018.10.008

20. Zhang C, Wang S, Liu Y, Yang C. Epigenetics in myeloid derived suppressor cells: a sheathed sword towards cancer. Oncotarget. (2016) 7:57452-63. doi: 10.18632/oncotarget.10767
21. Sandoval-Basilio J, Gonzalez-Gonzalez R, Bologna-Molina R, IsiordiaEspinoza M, Leija-Montoya G, Alcaraz-Estrada SL, et al. Epigenetic mechanisms in odontogenic tumors: a literature review. Arch Oral Biol. (2018) 87:211-7. doi: 10.1016/j.archoralbio.2017.12.029

22. El Gazzar M. microRNAs as potential regulators of myeloidderived suppressor cell expansion. Innate Immun. (2014) 20:227-38. doi: $10.1177 / 1753425913489850$

23. Shang W, Tang Z, Gao Y, Qi H, Su X, Zhang Y, et al. LncRNA RNCR3 promotes chop expression by sponging miR-185-5p during MDSC differentiation. Oncotarget. (2017) 8:111754-69. doi: $10.18632 /$ oncotarget.22906

24. Shang W, Gao Y, Tang Z, Zhang Y, Yang R. The pseudogene Olfr29-ps1 promotes the suppressive function and differentiation of monocytic MDSCs. Cancer Immunol Res. (2019) 7:813-27. doi: 10.1158/2326-6066.CIR-18-0443

25. Zheng Y, Tian X, Wang T, Xia X, Cao F, Tian J, et al. Long noncoding RNA Pvt1 regulates the immunosuppression activity of granulocytic myeloidderived suppressor cells in tumor-bearing mice. Mol Cancer. (2019) 18:61. doi: 10.1186/s12943-019-0978-2

26. Zhou Q, Tang X, Tian X, Tian J, Zhang Y, Ma J, et al. LncRNA MALAT1 negatively regulates MDSCs in patients with lung cancer. J Cancer. (2018) 9:2436-42. doi: 10.7150/jca.24796

27. Tian X, Ma J, Wang $\mathrm{T}$, Tian J, Zhang $\mathrm{Y}$, Mao L, et al. Long non-coding RNA HOXA transcript antisense RNA myeloid-specific 1HOXA1 axis downregulates the immunosuppressive activity of myeloidderived suppressor cells in lung cancer. Front Immunol. (2018) 9:473. doi: $10.3389 /$ fimmu.2018.00473

28. Tian X, Ma J, Wang T, Tian J, Zheng Y, Peng R, et al. Long non-coding RNA RUNXOR accelerates MDSC-mediated immunosuppression in lung cancer. BMC Cancer. (2018) 18:660. doi: 10.1186/s12885-018-4564-6

29. Ma X, Sheng S, Wu J, Jiang Y, Gao X, Cen X, et al. LncRNAs as an intermediate in HPV16 promoting myeloid-derived suppressor cell recruitment of head and neck squamous cell carcinoma. Oncotarget. (2017) 8:42061-75. doi: 10.18632/oncotarget.14939

30. Fujisaka Y, Iwata T, Tamai K, Nakamura M, Mochizuki M, Shibuya R, et al. Long non-coding RNA HOTAIR up-regulates chemokine (C-C motif) ligand 2 and promotes proliferation of macrophages and myeloid-derived suppressor cells in hepatocellular carcinoma cell lines. Oncol Lett. (2018) 15:509-14. doi: $10.3892 / 01.2017 .7322$

31. Wei S, Wang K. Long noncoding RNAs: pivotal regulators in acute myeloid leukemia. Exp Hematol Oncol. (2015) 5:30. doi: 10.1186/s40164-016-0059-9

32. Bhan A, Soleimani M, Mandal SS. Long noncoding RNA and cancer: a new paradigm. Cancer Res. (2017) 77:3965-81. doi: 10.1158/0008-5472.CAN-16-2634

33. Quinn JJ, Chang HY. Unique features of long non-coding RNA biogenesis and function. Nat Rev Genet. (2016) 17:47-62. doi: 10.1038/nrg.2015.10

34. Ayupe AC, Tahira AC, Camargo L, Beckedorff FC, Verjovski-Almeida S, Reis EM. Global analysis of biogenesis, stability and sub-cellular localization of lncRNAs mapping to intragenic regions of the human genome. RNA Biol. (2015) 12:877-92. doi: 10.1080/15476286.2015.1062960

35. Cabili MN, Trapnell C, Goff L, Koziol M, Tazon-Vega B, Regev A, et al. Integrative annotation of human large intergenic noncoding RNAs reveals global properties and specific subclasses. Genes Dev. (2011) 25:1915-27. doi: 10.1101/gad.17446611

36. Marchese FP, Raimondi I, Huarte M. The multidimensional mechanisms of long noncoding RNA function. Genome Biol. (2017) 18:206. doi: $10.1186 /$ s13059-017-1348-2

37. Anfossi S, Babayan A, Pantel K, Calin GA. Clinical utility of circulating non-coding RNAs-an update. Nat Rev Clin Oncol. (2018) 15:541-63. doi: 10.1038/s41571-018-0035-x

38. Balas MM, Johnson AM. Exploring the mechanisms behind long noncoding RNAs and cancer. Noncoding RNA Res. (2018) 3:108-17. doi: 10.1016/j.ncrna.2018.03.001

39. Mathy NW, Chen XM. Long non-coding RNAs (lncRNAs) and their transcriptional control of inflammatory responses. J Biol Chem. (2017) 292:12375-82. doi: 10.1074/jbc.R116.760884

40. Tian X, Tian J, Tang X, Ma J, Wang S. Long non-coding RNAs in the regulation of myeloid cells. J Hematol Oncol. (2016) 9:99. doi: $10.1186 / \mathrm{s} 13045-016-0333-7$ 
41. Zhang L, Cao Y, Wei M, Jiang X, Jia D. Long noncoding RNA-RNCR3 overexpression deleteriously affects the growth of glioblastoma cells through miR-185-5p/Kruppel-like factor 16 axis. J Cell Biochem. (2018) 119:9081-9. doi: $10.1002 /$ jcb. 27167

42. Shan K, Jiang Q, Wang XQ, Wang YN, Yang H, Yao MD, et al. Role of long non-coding RNA-RNCR3 in atherosclerosis-related vascular dysfunction. Cell Death Dis. (2016) 7:e2248. doi: 10.1038/cddis. 2016.145

43. Shan K, Li CP, Liu C, Liu X, Yan B. RNCR3: a regulator of diabetes mellitusrelated retinal microvascular dysfunction. Biochem Biophys Res Commun. (2017) 482:777-83. doi: 10.1016/j.bbrc.2016.11.110

44. Tian C, Deng Y, Jin Y, Shi S, Bi H. Long non-coding RNA RNCR3 promotes prostate cancer progression through targeting miR-185-5p. Am J Transl Res. (2018) 10:1562-70.

45. Thevenot PT, Sierra RA, Raber PL, Al-Khami AA, Trillo-Tinoco J, Zarreii P, et al. The stress-response sensor chop regulates the function and accumulation of myeloid-derived suppressor cells in tumors. Immunity. (2014) 41:389-401. doi: 10.1016/j.immuni.2014.08.015

46. Colombo T, Farina L, Macino G, Paci P. PVT1: a rising star among oncogenic long noncoding RNAs. Biomed Res Int. (2015) 2015:304208. doi: 10.1155/2015/304208

47. Lu D, Luo P, Wang Q, Ye Y, Wang B. lncRNA PVT1 in cancer: a review and meta-analysis. Clin Chim Acta. (2017) 474:1-7. doi: 10.1016/j.cca.2017.08.038

48. Zhao M, Wang S, Li Q, Ji Q, Guo P, Liu X. MALAT1: a long non-coding RNA highly associated with human cancers. Oncol Lett. (2018) 16:19-26. doi: 10.3892/ol.2018.8613

49. Wan L, Kong J, Tang J, Wu Y, Xu E, Lai M, et al. HOTAIRM1 as a potential biomarker for diagnosis of colorectal cancer functions the role in the tumour suppressor. J Cell Mol Med. (2016) 20:2036-44. doi: 10.1111/ jcmm. 12892

50. Xin J, Li J, Feng Y, Wang L, Zhang Y, Yang R. Downregulation of long noncoding RNA HOTAIRM1 promotes monocyte/dendritic cell differentiation through competitively binding to endogenous miR-3960. Onco Targets Ther. (2017) 10:1307-15. doi: 10.2147/OTT.S124201

51. Wang H, Li W, Guo R, Sun J, Cui J, Wang G, et al. An intragenic long noncoding RNA interacts epigenetically with the RUNX1 promoter and enhancer chromatin DNA in hematopoietic malignancies. Int J Cancer. (2014) 135:2783-94. doi: 10.1002/ijc.28922

52. Tian J, Rui K, Tang X, Ma J, Wang Y, Tian X, et al. MicroRNA-9 regulates the differentiation and function of myeloid-derived suppressor cells via targeting Runx1. J Immunol. (2015) 195:1301-11. doi: 10.4049/jimmunol.1500209

53. Tang Q, Hann SS. HOTAIR: an oncogenic long non-coding RNA in human cancer. Cell Physiol Biochem. (2018) 47:893-913. doi: 10.1159/ 000490131
54. Yu A, Wang Y, Yin J, Zhang J, Cao S, Cao J, et al. Microarray analysis of long non-coding RNA expression profiles in monocytic myeloid-derived suppressor cells in Echinococcus granulosus-infected mice. Parasit Vectors. (2018) 11:327. doi: 10.1186/s13071-018-2905-6

55. Pan W, Zhou HJ, Shen YJ, Wang Y, Xu YX, Hu Y, et al. Surveillance on the status of immune cells after Echinnococcus granulosus protoscoleces infection in Balb/c mice. PLoS ONE. (2013) 8:e59746. doi: 10.1371/journal.pone.0059746

56. Leti F, DiStefano JK. Long noncoding RNAs as diagnostic and therapeutic targets in type 2 diabetes and related complications. Genes (Basel). (2017) 8:E207. doi: 10.3390/genes8080207

57. Sun Z, Yang S, Zhou Q, Wang G, Song J, Li Z, et al. Emerging role of exosomederived long non-coding RNAs in tumor microenvironment. Mol Cancer. (2018) 17:82. doi: 10.1186/s12943-018-0831-Z

58. Wang M, Zhou L, Yu F, Zhang Y, Li P, Wang K. The functional roles of exosomal long non-coding RNAs in cancer. Cell Mol Life Sci. (2019) 76:2059-76. doi: 10.1007/s00018-019-03018-3

59. Zoller M. Janus-faced myeloid-derived suppressor cell exosomes for the good and the bad in cancer and autoimmune disease. Front Immunol. (2018) 9:137. doi: 10.3389/fimmu.2018.00137

60. $\mathrm{Wu} \mathrm{R}$, Gao W, Yao K, Ge J. Roles of exosomes derived from immune cells in cardiovascular diseases. Front Immunol. (2019) 10:648. doi: 10.3389/fimmu.2019.00648

61. Shen M, Ren X. New insights into the biological impacts of immune cell-derived exosomes within the tumor environment. Cancer Lett. (2018) 431:115-22. doi: 10.1016/j.canlet.2018.05.040

62. Geis-Asteggiante L, Belew AT, Clements VK, Edwards NJ, OstrandRosenberg S, El-Sayed NM, et al. Differential content of proteins, mRNAs, and miRNAs suggests that MDSC and their exosomes may mediate distinct immune suppressive functions. J Proteome Res. (2018) 17:486-98. doi: 10.1021/acs.jproteome.7b00646

Conflict of Interest Statement: The authors declare that the research was conducted in the absence of any commercial or financial relationships that could be construed as a potential conflict of interest.

Copyright (c) 2019 Leija Montoya, González Ramírez, Sandoval Basilio, Serafín Higuera, Isiordia Espinoza, González González and Serafín Higuera. This is an open-access article distributed under the terms of the Creative Commons Attribution License (CC BY). The use, distribution or reproduction in other forums is permitted, provided the original author(s) and the copyright owner(s) are credited and that the original publication in this journal is cited, in accordance with accepted academic practice. No use, distribution or reproduction is permitted which does not comply with these terms. 